\title{
Que faire des déchets TFA issus des opérations de démantèlement des sites nucléaires ? Les sujets de débat
}

\author{
Jacques Repussard* \\ Ancien Directeur-Général de l'IRSN et Chairman de MELODI, 78860 St Nom la Bretèche, France.
}

Reçu le 7 juillet 2019 / Accepté le 15 juillet 2019

\begin{abstract}
Résumé - Les déchets TFA issus des opérations de démantèlement des sites nucléaires constituent une des questions importantes du grand débat national lancé par la Commission Nationale du Débat Public dans le cadre de la révision du Plan National de Gestion des Matières et Déchets Radioactifs. Dans leur définition actuelle, ces déchets TFA présentent des volumes considérables qui excèdent les capacités actuelles de stockage alors que les enjeux de radioprotection sont modestes par rapport à ceux posés par les déchets de haute activité à vie longue. Cet article analyse les tenants et aboutissants de la gestion des déchets TFA issus du démantèlement et propose une approche mesurée basée sur une analyse appropriée de l'ensemble des enjeux.
\end{abstract}

Mots clés : déchets radioactifs / TFA / faibles doses / radioprotection / PNGMDR

\begin{abstract}
What to do with very low level radioactive waste resulting from the dismantling of nuclear plants? Issues for debate. Very low activity radioactive wastes from nuclear site dismantling constitute one of the critical issues of the large national debate launched in France by the National Commission of Public Debate at time of the revision of the national plan of management of radioactive wastes and matters. The very low activity radioactive wastes, as currently defined, present considerable volumes that exceed the actual storage capacities while the radiological protection challenges are modest compared to those raised by high activity and long life waste. This article analyzes the ins and outs of the management of the very low activity radioactive wastes from dismantling and proposes a measured approach based on an appropriate analysis of all the challenges.
\end{abstract}

Keywords: radioactive waste / very low activity waste / low dose / radiological protection / PNGMDR

\section{Introduction}

Toute activité humaine (et plus largement même toute vie sur la planète) génère des matières sans usage ultérieur pour leur producteur, que le dictionnaire définit comme des «déchets». Selon les époques et le développement des sociétés humaines, ces déchets sont soit dispersés dans l'environnement, soit «stockés» dans des lieux dédiés, et parfois surveillés, le cas échéant après un traitement, voire une opération de recyclage en général motivée par la rareté de certaines matières. Certains de ces déchets comportent des substances toxiques (métaux, molécules chimiques, toxines...) qui dans le premier cas de figure se dispersent au fil du temps, ou dans le second cas que l'on accumule en surveillant les rejets toxiques vers le milieu ambiant, plus ou moins bien protégé en fonction des précautions prises pour limiter

\footnotetext{
* Auteur de correspondance : jrepussard@aol.com
}

l'exposition humaine et celle des écosystèmes. L'industrie nucléaire n'échappe pas à cette pratique, bien que généralement considérée comme davantage encadrée que d'autres activités. Les déchets radioactifs de toutes origines (industrielle, médicale, défense) sont en effet une préoccupation majeure au titre de la protection des hommes et de l'environnement contre les rayonnements ionisants (RI) (Bourguignon et al., 2017), car même si certains de ces déchets ne présentent qu'une faible radioactivité, l'exposition aux rayonnements ionisants qu'ils peuvent générer, pour la population aussi bien que pour les travailleurs concernés, doit être la plus réduite possible. Les installations nucléaires (notamment les réacteurs électronucléaires, mais aussi les services de médecine nucléaire des hôpitaux) sont autorisés à relâcher dans leur environnement proche (eaux de surface et atmosphère) des quantités limitées de «rejets» radioactifs liquides ou gazeux à toxicité réduite, après vérification de l'absence de risque d'accumulation de radionucléides au sein des écosystèmes, et de la quasi absence d'exposition humaine à 
ces rejets, au sein de la population environnante. Cette radioactivité relâchée dans l'environnement dans des conditions propres à optimiser la dispersion (hautes cheminées, choix des sites maritimes, rejets fluviaux contrôlés...) reste détectable à l'aide d'instruments suffisamment précis, et fait l'objet d'une surveillance permanente, à la fois par les exploitants nucléaires eux-mêmes, et par l'Institut de radioprotection et de sûreté nucléaire, qui dispose pour cela de stations de mesure indépendantes.

Par ailleurs, ces mêmes installations ont obligation d'organiser la traçabilité et la gestion dans le temps de tous les déchets radioactifs, autres que ceux-ci-dessus appelés « effluents», produits, conditionnés et éventuellement transformés, afin de maîtriser le risque d'exposition humaine et écologique au-delà des niveaux (très bas, et en particulier très inférieurs aux niveaux d'exposition radiologique d'origine naturelle, cosmique ou géologique) édictés par les règles générales en matière de radioprotection, issues du consensus scientifique international.

Une partie de ces déchets comporte en effet des niveaux de radioactivité potentiellement et durablement significatifs (déchets issus des laboratoires de recherche, activités de maintenance industrielle des installations nucléaires, etc...), voire extrêmement élevés (déchets issus des opérations de recyclage du combustible nucléaire par exemple) tels que des installations spécialement conçues doivent être construites pour les conditionner, les transporter puis les stocker de manière sûre et pérenne. Une autre partie de ces déchets présente des niveaux de radioactivité très bas, parfois du même ordre que certains matériaux naturellement radioactifs présents dans notre environnement habituel, comme le granite, et dont l'activité va décroître assez rapidement, en l'espace de quelques décennies. Ces déchets, dits TFA (de très faible activité), doivent selon les règles applicables en France, être stockés et surveillés dans des centres spécialisés gérés par l'Andra.

En outre, une interdiction de recyclage de matières susceptibles de comporter des radionucléides artificiels, en dehors d'opérations destinées à satisfaire des besoins internes à l'industrie nucléaire, résulte de fait en France (à la différence d'autres pays européens notamment) des disposition du Code de la Santé publique qui proscrivent l'incorporation de radionucléides artificiels dans les biens de consommation, sauf dérogation accordée par les Pouvoirs Publics.

Ainsi, au fur et à mesure de l'implantation d'une industrie nucléaire particulièrement présente sur l'ensemble du territoire national, un encadrement réglementaire exigeant des déchets produits par cette industrie a été mis en place, assurant efficacement l'absence de risque radiologique significatif pour la population et l'environnement, hors circonstances accidentelles toujours possibles bien que peu probables. Afin de réduire le plus possible la probabilité que des déchets radioactifs se trouvent en situation de générer une exposition radiologique de la population, la réglementation en vigueur prescrit que tout déchet généré dans les «zones» où sont présentes des sources radioactives, au sein d'une installation réglementée au regard du risque radiologique, doit être considéré comme déchet radioactif, sauf à ce que le producteur de ces déchets démontre l'absence de radioactivité artificielle. En pratique, cela conduit à transférer dans les centres Andra des déchets dont jusqu'à $50 \%$ ne comportent aucune radioactivité, le coût de ce mode de gestion des déchets restant inférieur au coût des contrôles à mettre en œuvre pour s'assurer avec certitude de l'absence de radioactivité artificielle dans une partie des déchets.

Cette situation concourt naturellement à l'acceptabilité de la présence de cette industrie sur le territoire, ce qui confère en retour au cadre législatif réglementaire une grande stabilité et aux pratiques de surveillance par l'État une obligation de haute performance et de transparence. De nombreuses instances officielles ont en outre été créées au fil du temps pour assurer l'accès à l'information et le dialogue sur le dispositif d'encadrement des déchets radioactifs, et sur ses évolutions, par exemple les commissions locales d'information (CLI) et le Haut Comité pour la transparence et l'information sur la sécurité nucléaire [HCTISN]).

Pourtant, deux questions nouvelles arrivent à l'ordre du jour d'un débat national initié par les Pouvoirs Publics (CNDP, 2018) en application de la loi du 28 juin 2006 (Legifrance, 2006) et de la révision du Plan National de gestion des matières et déchets radioactifs (PNGMDR), en vue d'y associer la société toute entière, et ce indépendamment de la question de l'avenir de la filière nucléaire française:

- comment gérer les déchets dits à Haute activité et à vie longue (HAVL), qui représentent l'essentiel de la radioactivité présente sur un site nucléaire à travers le combustible et autres matières nucléaires qui y sont produites ou entreposées? Leur maîtrise doit être assurée pour des durées allant bien au-delà de celles dont les sociétés humaines ont l'expérience, en pratique de l'ordre du siècle. C'est l'objet d'un autre article de ce numéro de Radioprotection (Mercadal, 2019);

- comment gérer les déchets de Très faible activité (TFA) issus du démantèlement à venir des grandes installations nucléaires, dont le volume attendu est sans commune mesure avec celui des déchets TFA générés au fil de l'activité ordinaire de ces installations, et dont les caractéristiques sont souvent différentes de ces derniers?

L'objectif du présent article est d'éclairer la seconde question, concernant la gestion des déchets TFA issus des opérations de démantèlement à venir des sites nucléaires, au travers des grands paradigmes d'intérêt pour la société contemporaine: le risque bien sûr, sous toutes ses formes, et les conditions de sa gestion efficace et équitable, mais aussi les enjeux écologiques associés à ces grandes opérations de démantèlement, comme le devenir des sites nucléaires et des matériaux issus de la déconstruction des installations.

\section{Les données de la problématique des déchets TFA issus du démantèlement}

\subsection{Caractéristiques essentielles des matières issues de la déconstruction des centrales nucléaires}

Le volume de déchets radioactifs TFA qui pourraient être issus du démantèlement de l'ensemble des installations nucléaires françaises actuelles est estimé à $2200000 \mathrm{~m}^{3}$ (hors traitement des sols potentiellement contaminés) (IRSN, 2016). Ce volume est à mettre en rapport avec la capacité actuelle de $650000 \mathrm{~m}^{3}$ de stockage des déchets de la filière TFA dans le 
Centre industriel de regroupement, d'entreposage et de Stockage (Cires) de l'Andra situé à Morvilliers dans le département de l'Aube. Cette inadéquation évidente a déjà conduit à indiquer la nécessité d'une recherche de proportionnalité des solutions de gestion des déchets radioactifs à leur dangerosité (Hoorelbeke 2018), y compris si besoin à travers la mise à disposition d'un nouveau site de stockage. Ce volume est aussi à comparer avec l'ordre de grandeur de $10000000 \mathrm{~m}^{3}$ de déchets conventionnels qui seront issus de la déconstruction des parties non nucléaires des seules centrales EDF : la possibilité de synergies dans le traitement industriel de tous ces déchets, tout en assurant la gestion du risque radiologique, représente donc un enjeu majeur.

La part prépondérante en volume des déchets TFA générés au cours du processus de démantèlement est issue, d'une part, de la déconstruction des bétons et, d'autre part, des métaux. À côté des fers à béton, circuits électriques, et autres composants métalliques faiblement radioactifs, les centaines de générateurs de vapeurs des centrales nucléaires et les diffuseurs de l'usine George Besse 1 (Eurodif) constituent de grands volumes d'aciers de haute qualité, et de grande valeur potentielle aux plans économique et écologique (en termes notamment d'économie de l'énergie nécessaire à la production de quantités équivalentes à partir de minerais).

Les deux sources de la radioactivité de ces déchets, la contamination et l'activation, sont à prendre en compte pour la conduite des travaux de déconstruction, puis pour la gestion ultérieure des matériaux car leurs caractéristiques sont différentes en termes de nature des radionucléides, et de procédés de traitement, surfacique ou en profondeur.

Dans cette perspective, les recherches opérationnelles se poursuivent pour optimiser les procédés de déconstruction, de manière à protéger efficacement les travailleurs, les riverains et l'environnement, ainsi qu'à limiter les volumes de déchets devant faire l'objet d'une gestion dans la durée à raison de leur caractère radioactif avéré.

\subsection{Les sujets de débat}

\subsubsection{Le devenir des sites nucléaires}

Bien que théoriquement envisageable, le «retour à la nature » de ces sites industriels paraît hors d'atteinte pour plusieurs raisons, dont les deux principales seraient d'une part la difficulté d'éliminer de manière suffisante toutes les sources de pollution (radioactive ou autre), et d'autre part la longue durée des opérations de démantèlement, qui conduit naturellement à rechercher un équilibre socio-économique viable pour ces sites, en termes d'emploi, et de reconversion industrielle. Dans ce contexte, il paraît légitime d'envisager que le débat national évoque la perspective d'une gestion sur leur site même de production de certains déchets TFA (gravats notamment).

\subsubsection{La réglementation}

L'encadrement réglementaire des installations nucléaires couvre aussi leur phase de démantèlement, jusqu'à élimination de toute source de radioactivité artificielle. Il en va de même pour ce qui concerne la gestion des déchets issus du démantèlement, quel que soit le niveau de radioactivité. Ainsi, l'ouverture par le Gouvernement d'un débat public sur les modalités de gestion des déchets TFA issus du démantèlement des sites nucléaires doit être considérée comme autorisant un questionnement sur les modalités d'encadrement réglementaire de ces déchets et des sites après démantèlement, faute de quoi l'enjeu du débat se restreint au sujet de la recherche de nouveaux sites destinés à accueillir les grandes quantités de déchets TFA générés par les opérations futures de démantèlement. Les dispositions existantes des Codes de l'Environnement et de la Santé publique offrent d'ores et déjà une gamme de solutions assez complète. Toutefois certains aménagements, dont la portée pourrait être limitée aux opérations de démantèlement final d'installations nucléaires afin de ne pas perturber un ensemble réglementaire qui a par ailleurs fait ses preuves, pourraient se révéler nécessaires à l'issue du débat, en vue de la mise en œuvre des solutions les plus consensuelles. Il pourrait notamment s'agir des dispositions qui encadrent certaines opérations et matériaux au titre de la protection contre les rayonnements ionisants alors même que l'absence de tout risque radiologique pourrait être démontrée. Ainsi, certains déchets issus de la déconstruction de «zones nucléaires » pourraient faire l'objet d'un contrôle radiologique poussé à l'instar de celui pratiqué à l'échelle industrielle pour le contrôle de la qualité radiologique des eaux potables. Ces déchets pourraient alors être légitimement traités comme déchets conventionnels.

\subsubsection{Le recyclage de matériaux valorisables}

La réutilisation des bétons de démantèlement, en dehors de sites nucléaires, sous forme de granulats (travaux d'infrastructure routière par exemple) paraît a priori peu attractive au plan économique, compte tenu notamment de la lourdeur des contrôles radiologiques qui seraient nécessaires pour s'assurer de l'absence de particules de radionucléides plus fortement radioactives qui auraient pu contaminer le béton dans certaines «zones» de l'installation nucléaire. En revanche, la problématique est différente pour les métaux, de plus grande valeur et présents dans de si importantes quantités que l'enjeu de leur éventuelle réutilisation s'impose de lui-même. Leur volume, en conditionnement standard de déchets TFA, est en effet du même ordre de grandeur que la capacité totale actuellement autorisée du site de stockage de l'Andra pour les déchets TFA (CIRES) Pour ces métaux, une décontamination surfacique poussée, suivie d'une fusion dans une installation dédiée (notamment dotée de filtres des rejets gazeux et d'une surveillance radiologique adaptée) associée à un contrôle radiologique systématique poussé permettrait la mise en place d'une filière de recyclage proposant des produits suffisamment contrôlés pour ne pas nécessiter de traçabilité au plan radiologique, et donc éligibles à l'ensemble des transformations industrielles et usages ultérieurs, au même titre que les produits provenant d'autres filières, notamment d'importation. Il est à noter au passage que la récupération, dans les filtres, des radionucléides initialement présents à la surface ou au sein des déchets métalliques permettrait d'assurer la gestion par l'Andra de ces déchets radioactifs ultimes dans de bonnes conditions, en raison de leur volume réduit.

\section{Discussion}

Il ne s'agit pas ici de conduire le débat, ni a fortiori d'en ébaucher de possibles conclusions, ce qui relève de la seule 
responsabilité collective des acteurs et parties prenantes du Débat. Il s'agit seulement d'identifier certains paramètres clé de la mise en discussion des questions essentielles évoquées ci-dessus, notamment au plan de l'éthique de la gestion du risque radiologique, de l'éthique des préoccupations environnementales plus générales, ainsi que du rôle des technologies qui peuvent (ou non) rendre certaines solutions techniquement crédibles et économiquement possibles.

\subsection{Le risque radiologique}

Le risque radiologique est bien sûr la première des préoccupations pour l'ensemble des parties prenantes. Pourtant le risque sanitaire (cancers notamment) induit par une exposition maîtrisée aux rayonnements émis par des déchets TFA est extrêmement faible par rapport à d'autres types d'exposition, notamment dues à la radioactivité naturelle. L'application du principe de précaution (l'évaluation directe d'un risque radiologique aussi faible est scientifiquement hors de portée) amène à considérer en première approximation le risque de cancer radio-induit comme proportionnel à la dose reçue. Or la recherche a montré que le risque additionnel de contracter un cancer pour un individu ayant reçu une exposition de 1000 millisieverts (une exposition très élevée, jamais rencontrée dans un contexte industriel hors accident nucléaire) était de l'ordre de $5 \%$. Les doses évoquées dans les dossiers d'évaluation des risques au titre des déchets TFA étant de l'ordre du micro-sievert par an au plus pour des riverains, la règle de proportionnalité conduit à estimer un risque additionnel de cancer de l'ordre de 0,00005\%. Cependant, ce raisonnement mathématique ne suffit pas à positionner la perception par le public du risque radiologique. Ce dernier a acquis au fil du siècle dernier une image de grande dangerosité, au moins lorsqu'il est lié à des applications nucléaires industrielles ou militaires, puisque les expositions médicales à la radioactivité, qui présentent des risques similaires sont, elles, associées à un bénéfice individuel. Cette perception sociétale, ainsi que la logique du principe de précaution évoqué ci-dessus conduisent donc à poursuivre les recherches afin de mieux comprendre les mécanismes biologiques ms en jeu par de très faibles niveaux d'exposition aux rayonnements ionisants. Depuis 2010, la communauté scientifique européenne a notamment accentué ses travaux sur les effets des faibles doses dans le cadre de la Plateforme de recherche européenne MELODI, au sein d'EURATOM (Repussard, 2017). Les progrès de la biologie fondamentale, et la disponibilité de nouveaux outils d'analyse numérique, notamment en génétique, permettent d'espérer au cours de ce siècle un éclairage opérationnel sur les risques réellement associés à de telles expositions, en particulier compte tenu de la sensibilité aux rayonnements ionisants, dont on sait qu'elle varie d'un individu à l'autre (Foray et al., 2016).

Reflétant l'état actuel des connaissances scientifiques, les standards internationaux en matière de radioprotection, applicables aussi en France, imposent donc logiquement de réduire autant que possible en pratique les expositions aux rayonnements ionisants, même les plus faibles. Cette démarche impose de chercher à évaluer les risques d'exposition pour les groupes de personnes les plus exposées en raison des processus de gestion des déchets, au sein du public (riverains) et au sein des travailleurs. Il résulte de ces évaluations le choix de méthodes de démantèlement et de gestion des déchets produits (transformation, transport, stockage; protection des travailleurs contre l'exposition aux poussières par exemple) appropriées.

Ainsi, il serait possible de comparer, en termes d'impact radiologique, différents scénarios de référence pour le démantèlement, par exemple un scénario avec évacuation de l'ensemble des déchets susceptibles de présenter un risque radiologique à raison de leur provenance (zones nucléaires) vers un site Andra dédié (pratique actuelle de gestion des TFA) (Hoorelbeke, 2018), et un ou plusieurs scénarios prévoyant le maintien sur place des déchets de béton très peu ou pas du tout radioactifs, et la transformation des métaux valorisables.

\subsection{La hiérarchisation des différents risques}

La préoccupation légitime du risque radiologique ne doit pas masquer la présence d'autres risques liés aux opérations de démantèlement, et notamment de production et de gestion des déchets TFA. Les principales sources d'autres risques concernent surtout les travailleurs (accidents classiques du travail, expositions à des conditions de travail difficiles (température, atmosphère contrôlée...) et à d'autres substances toxiques comme l'amiante... S'agissant des riverains (des sites en démantèlement, des voies de transport et des sites de stockage de déchets) et des membres du public plus généralement, les risques sont faibles, mais doivent être recensés et comparés selon les scénarios : exposition aux poussières, accidents de la route résultant $\mathrm{du}$ transport sur plusieurs années de très grandes quantités de matériaux, aspects sociaux et politiques le cas échéant (notamment dans le contexte de la création d'un nouveau site national de stockage de déchets, dont la création pourrait se révéler mal justifiée compte tenu de l'existence d'autres options de gestion, et donc difficilement acceptable).

\subsection{L'équité}

La comparaison technique des différents scénarios envisageables de démantèlement et de gestion des déchets TFA produits permettra d'optimiser la gestion de l'ensemble des risques, parmi lesquels le risque radiologique n'est pas nécessairement le paramètre pivot. Elle permettra de mettre en évidence les avantages et inconvénients des différents scénarios techniquement et économiquement envisageables.

Mais cette dimension d'analyse restera sans doute insuffisante au regard de la charge sociétale importante associée aux enjeux des déchets radioactifs, et en arrière-plan du devenir de l'industrie nucléaire. C'est pourquoi au-delà des critères techniques, l'équité perçue de la solution finalement retenue sera un paramètre essentiel de l'acceptabilité de cette solution, voire un premier pas vers un dialogue sociétal débarrassé de quelques lourds préjugés sur la question nucléaire.

Prenons un exemple concret: un site de stockage de déchets radioactifs de haute et moyenne activité, a été construit dans le roc granitique, en dessous du niveau de la mer, à Olkiluoto (Finlande) à proximité de celui du chantier de l'EPR, mais aussi à proximité de la ville du même nom, stockage dont les résidents avaient accepté le projet. Deux arguments avaient en effet suffi à générer un consensus suffisant à l'échelle locale : un dossier technique solide et bien évalué au plan du risque radiologique par l'Autorité de sureté nucléaire nationale, et aussi 
(et peut-être surtout ?) le caractère équitable du projet. En effet, il fut observé que la communauté d'Olkiluoto avait bénéficié depuis des décennies des retombées économiques très positives de la centrale nucléaire (comportant deux réacteurs électronucléaires, en plus de l'EPR en voie d'achèvement), et qu'il y avait donc une certaine logique à ce que les déchets produits par cette centrale soient gérés sur place ou presque, puisque la géologie le permettait de manière sûre.

La Finlande n'est pas la France, mais elle n'est pas dépourvue pour autant d'organisations non gouvernementales militant contre le déploiement de l'industrie nucléaire.

Cet exemple illustre une réalité qui existe aussi dans notre pays. Les citoyens sont très sensibles à l'équité des mesures d'aménagement proposées au plan national ou local, et la dimension technique (technocratique) des dossiers proposés ne suffit pas à bien représenter les enjeux. Ainsi le Débat National sur le devenir des déchets radioactifs porte une opportunité d'aborder explicitement cette dimension complémentaire essentielle au consensus. L'équité, comme la confiance, ne se décrète pas (Mercadal, 2019). Elle se construit pas à pas, en consultation avec les parties prenantes. Ainsi, la question de l'opportunité de construire un nouveau site national de stockage TFA, versus le choix de limiter les quantités de déchets déplacés en optimisant leur gestion sur les sites d'origine peut très bien être abordée sous cet angle, l'éclairage du risque ne suffisant sans doute pas à disqualifier l'une des options.

\subsection{Les enjeux environnementaux et écologiques}

Enfin, au même niveau que cette préoccupation d'équité se situe sans doute une attente sociétale croissante en termes d'impact environnemental et écologique des options envisageables pour les déchets TFA. Bien entendu les risques pour les écosystèmes, radiologique et autres, font partie des critères d'acceptabilité réglementaire des dossiers de projet. Mais il existe une autre dimension, non encore prise en compte dans l'approche réglementaire, mais répondant à une attente sociétale croissante, celle de l'impact du démantèlement en termes d'énergie dépensée ou économisée, de gestion des matières premières. Ainsi, le transport par voie routière de grandes quantités de matériaux acquiert-t-il une toute autre dimension que celle de la sûreté radiologique (sans réel enjeu) desdits transports. Ou encore la perspective du non réemploi d'immenses quantités d'aciers de haute qualité et ne présentant pas de risque radiologique après traitement peut paraître contraire au principe de gestion responsable des ressources, à raison tant de la non-utilisation de ressources en fait réutilisables, que du coût considérable pour la société du stockage dans des sites gérés par l'Andra de matières sans dangerosité radiologique (Mercat et Lamouroux, 2018). Le devenir des sites nucléaires, post-démantèlement, peut également s'analyser sous cet angle écologique au sens global du terme. En faisant apparaître, peut-être, de nouveaux arguments propres à orienter ses conclusions, le Débat National s'enrichirait sans doute d'entrer dans ces considérations, sur la base de dossiers et points de vue techniquement étayés.

\section{Conclusion}

Au-delà de ses enjeux techniques, portant notamment sur le devenir des déchets radioactifs de très faible activité, le Débat National sur la gestion des déchets radioactifs organisé par la CNDP constitue une opportunité de premier plan pour entamer la recherche d'un nouveau consensus national sur l'avenir de l'industrie électronucléaire, source précieuse d'énergie décarbonée, mais accablée par de nombreux problèmes issus pour partie d'une gestion trop déconnectée de la nation. Encore faut-il pour cela que les dossiers proposés analysent de manière appropriée les enjeux sous toutes leurs facettes et que l'ensemble des options envisageables soient abordées sans la censure des pétitions de principe simplistes, ni celle d'une réglementation qui n'a pas été conçue avec comme première préoccupation celle d'encadrer de manière optimale le processus de démantèlement massif des grands sites nucléaires. Cette réglementation, de son côté, devrait donc aussi retenir les conclusions du débat pour s'adapter aux choix de société, en veillant à ce que leur mise en œuvre soit exécutable dans le respect des principes généraux d'une gestion des risques de toute nature, et en veillant à l'usage optimal des ressources de la nation.

\section{Références}

Bourguignon M, Bérard P, Bertho JM, Farah J, Mercat C. 2017. What's next in Radioprotection? Radioprotection 52(1): 21-28. DOI: $10.1051 /$ radiopro/2017007.

CNDP 2018. Cinquième édition du plan national de gestion des matières et déchets radioactifs. Décision CNDP $N^{\circ} 2018 / 25 /$ PNGMDR/1. https://www.debatpublic.fr/plan-national-gestionmatieres-dechets-radioactifs-pngmdr.

Foray N, Bourguignon M, Hamada N. 2016. Individual response to ionizing radiation. Mut. Res. 770: 369-386.

Hoorelbeke JM. 2018. De la nécessité d'une recherche de proportionnalité des solutions de gestion des déchets radioactifs à leur dangerosité. Radioprotection 53(3): 167-173. DOI: 10.1051/radiopro/2018028.

IRSN. 2016. Déchets radioactifs de très faible activité : la doctrine doit-elle évoluer? Rapport IRS N/DG/2016-00002.

Legifrance 2006. Loi n ${ }^{\circ} 2006-739$ du 28 juin 2006 de programme relative à la gestion durable des matières et déchets radioactifs (modifiée par la loi $\mathrm{n}^{\circ} 2016-1015$ du 25 juillet 2016).

Mercadal G. 2019. La gestion des déchets radioactifs à vie longue : confiés à l'argile ou bien gérés par la société? Radioprotection 54(3): 169-173.

Mercat C, Lamouroux C. 2018. Évaluation de stratégies de gestion des déchets faiblement radioactifs au regard des critères MTD «meilleure technique disponible». Radioprotection 53(4): 249-254. DOI: 10.1051/radiopro/2018035.

Repussard J. 2017. Low dose effects research in Europe: eight years of evolution towards new paradigms. Radioprotection 52(4): 251-258. DOI: 10.1051/radiopro/20170352.

Citation de l'article : Repussard J. 2019. Que faire des déchets TFA issus des opérations de démantèlement des sites nucléaires ? Les sujets de débat. Radioprotection 54(3): 163-167 